\title{
LO-NORM-BASED SPARSE REPRESENTATION THROUGH ALTERNATE PROJECTIONS
}

\author{
Luis Mancera, Javier Portilla* \\ Visual Information Processing Group \\ Department of Computer Science and Artificial Intelligence \\ Universidad de Granada \\ \{mancera, javier\}@ decsai.ugr.es
}

We present a simple and robust method for finding sparse representations in overcomplete transforms, based on minimization of the L0-norm. Our method is better than current solutions based on minimization of the L1-norm in terms of energy compaction. These results strongly question the equivalence of minimizing both norms in real conditions. We also show application to in-painting (interpolation of lost pixels).

Index Terms - Image representation, restoration.

\section{INTRODUCTION AND IMAGE MODEL}

Overcomplete representations allow for a more powerful image analysis and processing compared to critically sampled ones, because they favor the extraction of relevant local features $[1,2]$ and they also allow for invariance to translation, rotation, phase, etc. $[3,4]$. So, it is not surprising that the human visual representation is highly redundant [5]. In addition, several studies indicate that the proportion of neurons in the visual system responding simultaneously is small [2]. This seems an efficient way of storing and processing the information. Therefore, in image processing we are interested in sparse overcomplete representations, that is, approximating the images as linear combinations of few elementary functions from a large dictionary $[6,7,2,8]$. Sparseness has been used, associated to high-order moments, as a figure of merit for reducing mutual information among responses (e.g., [9]). Most degradation sources decrease the sparseness of the wavelet coefficients (e.g., $[10,11])$, and thus we can compensate for part of the degradation by finding sparse approximations to the observations $[12,13]$. For instance, we can fill-in missing pixels by imposing that the resulting image is sparse in a suitable representation [14].

In an overcomplete linear system, the level of sparseness depends on: 1) the set of elementary functions available for the representation; 2) the non-linear selection mechanism of elementary functions; and 3) the statistical properties of the input. In this work we use the Curvelet pyramid [15], a tight frame based on ridge functions. We have seen that it provides

\footnotetext{
*Both authors funded by grant TIC2003-1504 from the Ministerio de Ciencia y Tecnologia. JP is under the "Ramon y Cajal "program.
}

good compaction results compared to other tight frames. We propose a simple and robust coefficient selection mechanism based on minimizing the number of non-zeros and compare it with methods based on minimizing the sum of absolute values. We also show application to the in-painting problem.

\section{THE SPARSE REPRESENTATION PROBLEM}

Let's consider a $N \times M$ matrix $\boldsymbol{\Phi}$ with $M>N$ and $\operatorname{rank}(\boldsymbol{\Phi})=$ $N$. Then, for an observation $\mathbf{x} \in \mathbb{R}^{N}$ the system of equations:

$$
\Phi \mathbf{a}=\mathbf{x},
$$

has infinite solutions in $\mathbf{a} \in \mathbb{R}^{M}$. The minimum L2-norm solution, $\mathbf{a}_{L S}=\mathbf{\Psi}_{\mathbf{X}}$, is often chosen, where $\boldsymbol{\Psi}=\boldsymbol{\Phi}^{T}\left[\boldsymbol{\Phi} \boldsymbol{\Phi}^{T}\right]^{-1}$ is the pseudoinverse of $\boldsymbol{\Phi}$ (note that it corresponds to the analysis operator). This is computationally simple, specially when $\boldsymbol{\Phi}$ is self-invertible ( $\boldsymbol{\Psi}=\boldsymbol{\Phi}^{T}$ ). Unfortunately, it is clearly not the best option in terms of efficiency (in storage and processing) and independence among coefficients. A more interesting alternative is to solve the following problem:

$$
\hat{\mathbf{a}}_{0 H}=\arg \min _{\mathbf{a}}\|\mathbf{a}\|_{0} \text { s.t. } \Phi \mathbf{a}=\mathbf{x} .
$$

where $\|\mathbf{a}\|_{0}$ is the $\mathrm{L} 0$-norm of a vector $\mathbf{a}$, i.e, the number of its non-zero coefficients. In many practical situations it is convenient to allow for a certain degree of error, and we can relax the optimization constraint using the following formulation:

$$
\hat{\mathbf{a}}_{0 S}=\arg \min _{\mathbf{a}}\left\{\|\mathbf{a}\|_{0}+\lambda\|\mathbf{\Phi} \mathbf{a}-\mathbf{x}\|_{2}^{2}\right\}
$$

where $\lambda \in \mathbb{R}^{+}$. Unfortunately, the only known method to solve these equations exactly is combinatorial, thus NP- complex [16]. To overcome this problem, the following alternative formulation has been proposed [8]:

$$
\begin{array}{r}
\hat{\mathbf{a}}_{1 H}=\arg \min _{\mathbf{a}}\|\mathbf{a}\|_{1} \text { s.t. } \mathbf{\Phi} \mathbf{a}=\mathbf{x} . \\
\hat{\mathbf{a}}_{1 S}=\arg \min _{\mathbf{a}}\left\{\|\mathbf{a}\|_{1}+\lambda\|\mathbf{\Phi} \mathbf{a}-\mathbf{x}\|_{2}^{2}\right\}
\end{array}
$$

where $\|\mathbf{a}\|_{1}=\sum_{i=1}^{M}\left|a_{i}\right|$ denotes the L1-norm of vector $\mathbf{a}$. Basis Pursuit (BP) and Basis Pursuit Denoising (BPDN) [8] 
make use of the convexity of the objective functions in Eqs. (4) and (5) to find optimal solutions using linear programming. However, when dealing with large vectors, these methods are computationally very expensive [17]. Recently, an efficient method achieving low L1-norm representations [17] has been successfully applied to several applications such as in-painting and blind source separation. Though originally conceived to separate different morphological aspects of an image by using multiple dictionaries with complementary features, it is easily adapted for obtaining sparse representations in a single dictionary (called here Single Dictionary MCA, SDMCA).

Sufficient conditions of equivalence of using L1 or L0norm as objective function given in [18] requires the proportion of non-zero coefficients to be extremely small, having thus little practical application [16]. More recently, the equivalence condition has been found to be a number of non-zeros proportional to $N$ [16]. However, this proportionality factor is difficult to calculate in general, and it is still not clear that equivalence conditions may hold using typical images and representations for useful sparseness levels (see, e.g., [19]).

\section{LO-NORM-BASED SPARSE REPRESENTATION}

In this section, we present a sub-optimal method for solving Eq. (3) that, being extremely simple, is reasonably efficient and improves the results given by L1-norm based methods in terms of compaction of energy in few non-zero coefficients and application to in-painting (see Section 4).

Note that solving Eq. (3) for a given $\left\|\hat{\mathbf{a}}_{0 S}\right\|_{0}=K$ is equivalent to solve it for a certain $\lambda$ value. So, we can reformulate Eq. (3) as looking for the vector a with $K$ non-zero coefficients minimizing the Euclidean distance to $\mathbf{x}$. That is, if $C(K)=\left\{\mathbf{a} \in \mathbb{R}^{M}:\|\mathbf{a}\|_{0}=K\right\}$, then:

$$
\hat{\mathbf{a}}(K)=\arg \min _{\mathbf{a} \in C(K)}\|\mathbf{\Phi} \mathbf{a}-\mathbf{x}\|_{2}
$$

To solve $\hat{\mathbf{a}}(K)$, we use both $C(K)$ and the affine set of solutions to Eq. (1), denoted $S(\boldsymbol{\Phi}, \mathbf{x})$. The latter is convex, but the former is not. Nevertheless, we apply the alternating projections method [20], which converges to a solution in $C(K)$ that is a local minimizer of the distance to $S(\boldsymbol{\Phi}, \mathbf{x})$, and thus of the distance between the sets in the image domain, as it can be easily proved provided that $\Psi$ is a tight frame. Let $P_{S(\boldsymbol{\Phi , \mathbf { x }})}^{\perp}(\mathbf{a})=\mathbf{a}+\mathbf{a}_{L S}-\boldsymbol{\Psi} \boldsymbol{\Phi} \mathbf{a}$ be the orthogonal projection of a onto $S(\mathbf{\Phi}, \mathbf{x})$. Then $\left\|\mathbf{a}-P_{S(\mathbf{\Phi}, \mathbf{x})}^{\perp}(\mathbf{a})\right\|_{2}=$ $\|\mathbf{\Psi}(\mathbf{x}-\mathbf{\Phi} \mathbf{a})\|_{2}=k\|\mathbf{x}-\mathbf{\Phi} \mathbf{a}\|_{2}$, with $k>0$. If $\hat{\mathbf{a}}(K) \in C(K)$ is a local minimizer of the distance to $S(\boldsymbol{\Phi}, \mathbf{x})$, then exists $\delta>0$ such that for every $\mathbf{a} \in C(K)$, if $\|\mathbf{a}-\hat{\mathbf{a}}(K)\|_{2}<\delta$, then $\left\|\mathbf{a}-P_{S(\boldsymbol{\Phi}, \mathbf{x})}^{\perp}(\mathbf{a})\right\|_{2} \geq\left\|\hat{\mathbf{a}}(K)-P_{S(\boldsymbol{\Phi}, \mathbf{x})}^{\perp}(\hat{\mathbf{a}}(K))\right\|_{2}$, thus $\|\mathbf{x}-\boldsymbol{\Phi} \mathbf{a}\|_{2} \geq\|\mathbf{x}-\boldsymbol{\Phi} \hat{\mathbf{a}}(K)\|_{2}$. That is, $\boldsymbol{\Phi} \hat{\mathbf{a}}(K)$ is a local minimizer in $\mathbf{a} \in C(K)$ of the Euclidean distance to $\mathbf{x}$ (q.e.d).

We note $h t(\mathbf{a}, K)$ the minimum Euclidean distance projection onto the set $C(K)$ of a vector $\mathbf{a}$, which is just the hard-thresholding operation preserving the $K$ greatest values in amplitude. Then, our method can be formulated as follows, given an initial $\mathbf{a}^{(0)}=h t\left(\mathbf{a}_{L S}, K\right)$ :

$$
\mathbf{a}^{(n+1)}=h t\left(P_{S(\boldsymbol{\Phi}, \mathbf{x})}^{\perp}\left(\mathbf{a}^{(n)}\right), K\right) .
$$

Iterations end when $\left\|\mathbf{a}^{(n+1)}-\mathbf{a}^{(n)}\right\|_{2}<\epsilon$. Fig. 1 illustrates our method with $K=1$. $A$ is the subspace spanned by the columns of $\Psi$.

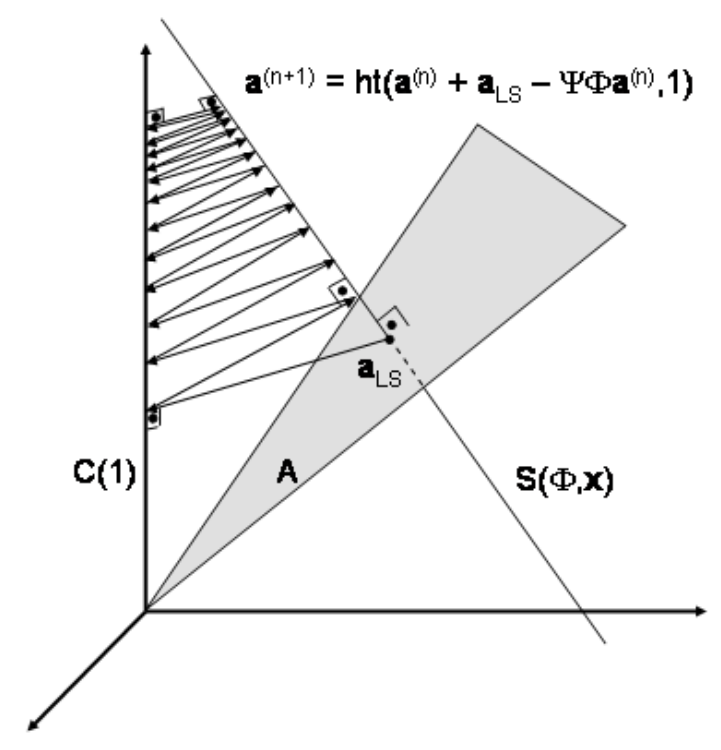

Fig. 1. L0-norm minimization through alternate projections.

\section{RESULTS AND DISCUSSION}

We have tested our method on a set of five 8-bit $256^{2}$ grayscale standard images (Boat, Einstein, Barbara, Peppers, Lena) ${ }^{1}$. We used CurveLab 2.0, a Curvelet implementation for MATLAB B (http://www.curvelet.org), with 5 scales. Redundancy factor is $\approx 7.2$. Basis Pursuit is implemented in Atomizer802 (http://www-stat.stanford.edu/ atomizer). We use Peak Signalto-Noise Ratio (PSNR) for comparing images, expressed as $10 \cdot \log _{10}\left(255^{2} / M S E\right)$ where $M S E$ is the mean square error. L0-norm is expressed as percentage of non-zero coefficients.

\subsection{Signal compaction}

Fig. 2 shows averaged L0-norm vs. PSNR of our method (solid line), BP (dotted line) and SDMCA (dashed line) for our set of test images. To obtain optimal performance of SDMCA, initial threshold has been set to the maximum amplitude value of the coefficients, and we have used a very large number of iterations (1000), same as for our method ${ }^{2}$. As solutions from BP and SDMCA are not strictly sparse, we have

\footnotetext{
${ }^{1}$ Boat and Barbara are cropped to $256^{2}$ starting at pixel $(200,100)$ and $(150,50)$ respectively. The black border surrounding Einstein has been removed by repeating the neighbor line.

${ }^{2}$ Much fewer iterations suffice in practical implementations.
} 
applied a threshold a posteriori to the coefficients in order to compare the methods, matching the number of non-zero coefficients of our solution. The curve obtained directly by thresholding $\mathbf{a}_{L S}$ has been also included as a reference (dasheddotted line). The objective of both BP and SDMCA is to solve Eq. (4), but our method approximates solutions to Eq. (3). As such, it would be more directly comparable with BPDN. However, we have obtained better results with the a posteriori threshold of BP than with BPDN. Our sub-optimal, simple method performs better than both SDMCA and BP. Thus, $\mathrm{BP}$ is not minimizing the $\mathrm{L} 0$-norm, implying that equivalence conditions of L0-L1 minimization are not met. As a more demanding comparison, we have LS-optimized the selected coefficients for each case, and only BP slightly surpassed our performance in the intermediate L0-norm range (graph not shown here). The vertical bold line indicates the percentage associated to $N$, for which value an optimal method would provide perfect reconstruction. This makes evident the suboptimal nature of all four compared methods (ours being the best). For these experiments, the computational cost of our method and SDMCA are similar, but BP is around 6 times slower. A visual example is shown in Fig. 3. Left is absolute error of the BP 2.1\%-approximation to Lena, cropped to $128^{2}$ for visibility. Right is our approximation with the same L0-norm, which obtains $2.37 \mathrm{dBs}$ better reconstruction.

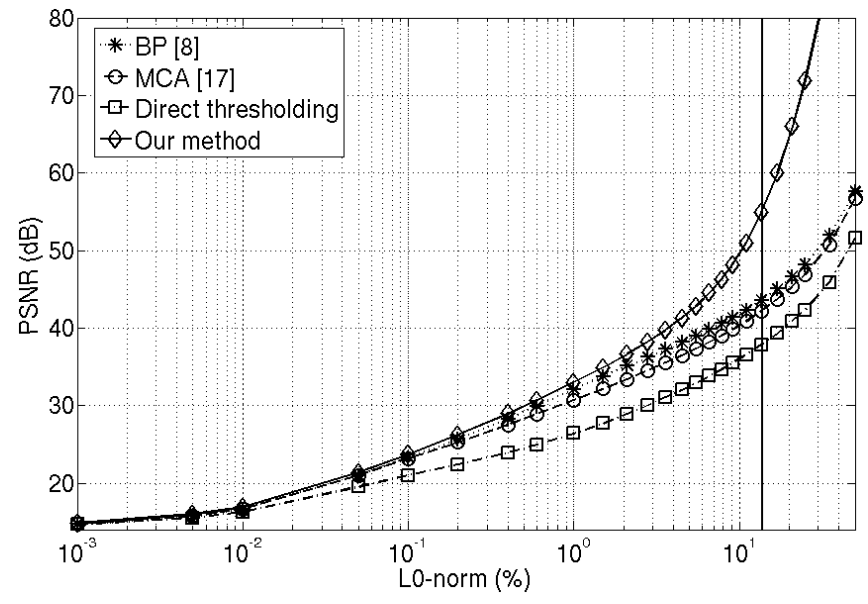

Fig. 2. Semi-logarithmic plot of averaged energy compaction results in our set of test images.

\subsection{Sparseness representation for in-painting}

When we have some lost pixels in an image, we can use the fact that typical images can be approximated as linear combination of relatively small subsets of features from a dictionary, to interpolate the missing data (in-painting). Given a sparseness level $K$ and a dictionary $\boldsymbol{\Phi}$, we look for the image sharing the set of observed pixels and having minimum Euclidean distance to the set of $K$-sparse images. In prac-

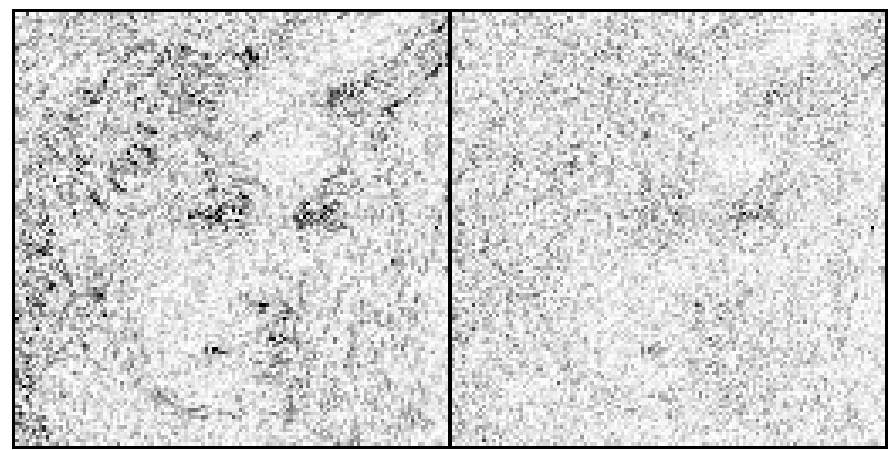

Fig. 3. Left, absolute error for BP approximation to Lena with $2.1 \%$ of coefficients ( $34.28 \mathrm{dBs})$. Black pixels correspond to high error. Right, our result with same L0-norm (36.65 dBs)

tice, the quality of the interpolation depends on having a good estimate of the $K$ providing the minimum mean-square error (MSE), which we note $K_{\text {opt }}$ (see paragraph below). We denote $\mathbf{x}$ the original image and $\mathbf{D}$ a diagonal matrix such that $d_{i, i}=1$ if $x_{i}$ is known and 0 otherwise. We also denote $\mathbf{a}_{L S}=\boldsymbol{\Psi D} \mathbf{x}$. Finally, we define $S_{\mathbf{D}}(\boldsymbol{\Phi}, \mathbf{x})$ as the set of vectors with perfect reconstruction of the observed pixels. The orthogonal projection operator onto $S_{\mathbf{D}}(\boldsymbol{\Phi}, \mathbf{x})$ is $P_{S_{\mathbf{D}}(\boldsymbol{\Phi}, \mathbf{x})}^{\perp}(\mathbf{a})=\mathbf{a}+\mathbf{a}_{L S}-\boldsymbol{\Psi D} \Phi \mathbf{a}$. Then, given $\mathbf{a}^{(0)}=\mathbf{a}_{L S}$, we obtain $\mathbf{a}^{(n+1)}=P_{S_{\mathbf{D}}(\boldsymbol{\Phi}, \mathbf{x})}^{\perp}\left(h t\left(\mathbf{a}^{(n)}, K\right)\right)$. Iterations end when $\left\|\mathbf{a}^{(n+1)}-\mathbf{a}^{(n)}\right\|_{2}<\epsilon$. Once converged, final estimation is $\hat{\mathbf{x}}=\mathbf{\Phi} \mathbf{a}^{(e n d)}$.

For the choice of $K$, we propose a method based on maximizing the mean-square value (MSV) of the interpolated pixels. For small values of $K$, only the most salient features of $\mathrm{x}$ are represented, and a low MSV results. When we choose a very high $K$, broken features are represented more accurately using many separated elementary functions than approximating them as parts of fewer features. This results in a poor interpolation and a low MSV of the estimated missing pixels. When using intermediate $K$ values, there are enough elementary functions to represent all relevant features, but not enough to describe finely the broken features, so missing pixels are filled-in with elementary functions, and a higher MSV results. Solid line (left axis) of Fig. 4 shows normalized MSE at estimated pixels w.r.t. $K$, where $K$ is normalized to $K_{\text {opt }}$. Dashed line shows normalized MSV. Dotted lines indicate standard deviation for each $K$. Dashed-dotted line is the real MSV of missing pixels of the original image, an upper bound of the MSV curve. Results are averaged for our set of test images randomly missing $40 \%$ of the pixels. For each test, 250 iterations of the method are executed. We set $\hat{K}_{\text {opt }}=\frac{1}{0.7} K_{\text {max }}$. Left panel of Fig. 5 shows the Barbara (cropped to $128^{2}$ ) result from SDMCA, running 100 iterations and setting the initial threshold below $10 \%$ of the coefficients (29.24 dBs). Right panel is our result using $\hat{K}_{\text {opt }}$ (100 iterations, $32.49 \mathrm{dBs}$ ). Better results are reported by adding some 
heuristics to a sparseness-based approach [14].

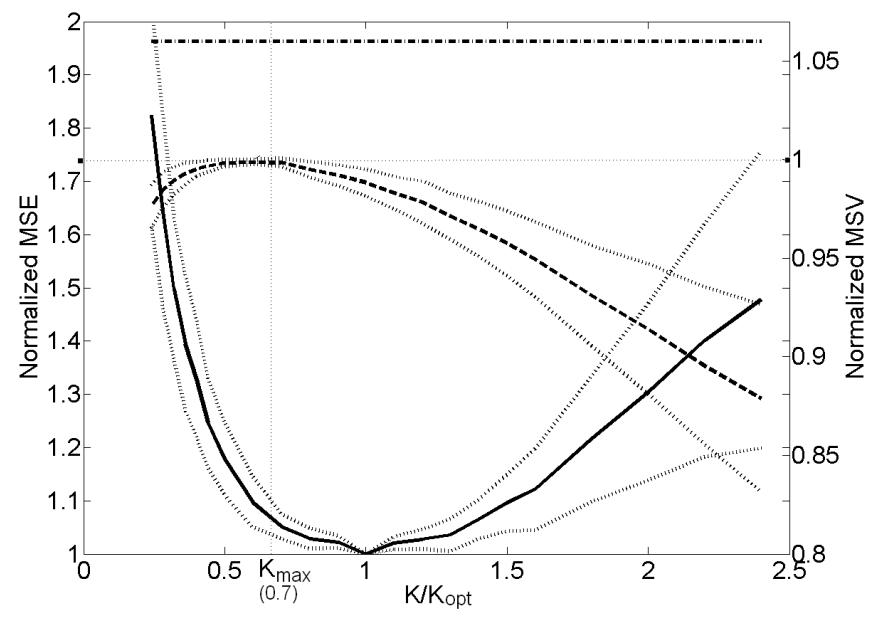

Fig. 4. Solid, normalized MS-error of estimated vs. real pixels (left axis) w.r.t. normalized values of $K$ averaged in our set of test images for a degradation randomly missing $40 \%$ of pixels. Dashed, normalized MS-value of estimated pixels (right axis). Dotted, standard deviation for each K. Dasheddotted, real MS-value.

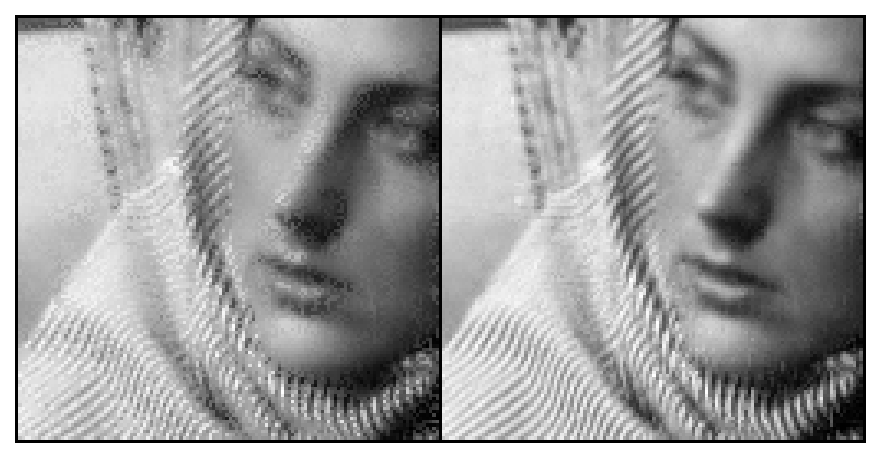

Fig. 5. Left, using SDMCA for a $40 \%$ missing pixels observation (29.24 dBs). Right, our result (32.49 dBs).

\section{CONCLUSIONS}

Ours is, to the best of our knowledge, the first practical method based on minimizing directly the L0-norm of the representation. We have compared it with optimal (BP) and suboptimal (SDMCA) solutions minimizing the L1-norm, in advantageous conditions for them. Our method is better in terms of compaction of energy in few non-zero coefficients, and similar to SDMCA in computational cost (BP is much slower). These results question the equivalence of minimizing L1 and L0-norm in practice. For in-painting application, our method provides a remarkably better estimation than practical L1norm-based method. In the future, we will develop more powerful minimization strategies and extend to other applications.

\section{REFERENCES}

[1] E.P. Simoncelli, W.T. Freeman, E.H. Adelson, D.J. Heeger. Shiftable Multi-Scale Transforms. IEEE Trans. Inf. Theory, 38, 2, 587-607, 1992.

[2] B.A. Olshausen, D.J. Field. Sparse Coding of Sensory Inputs. Curr. Opinion in Neurobiology, 14, 481-87, 2004.

[3] R.R. Coifman, D.L. Donoho. Translation Invariant De-noising. Lecture Notes in Statistics, 103, 125-50, 1995.

[4] E. Candes, J. Romberg, T. Tao. Stable Signal Recovery from Incomplete and Innacurate Measurements. Proc. of the SPIE, 5914, San Diego, CA, Aug. 2005.

[5] B.A. Wandell. Foundations of Vision. Sinauer, 1995.

[6] S.G. Mallat. A Theory for multiresolution signal decomposition: The wavelet representation. PAMI, 11, pp. 674-693, 1989.

[7] H. Barlow. Redundancy Reduction Revisited. Network: Computation in Neural Systems, 12, pp. 241-253, 2001.

[8] S.S. Chen, D.L. Donoho, M.A. Saunders. Atomic Decomposition by Basis Pursuit. SIAM, 20, 1, pp. 33-61, 1998.

[9] P. Comon. Independent Component Analysis. A New Concept?. Signal Processing, 36, pp. 287-314, 1994.

[10] F. Rooms, W. Philips, J. Portilla. Parametric PSF Estimation via Sparseness maximization in the wavelet domain. IEEE Trans. Image Proc., 12, 11, pp. 1338-1351, 2003.

[11] Z. Wang, G. Wu, H.R. Sheikh, E.P. Simoncelli, E.H. Yang, A.C. Bovik. Quality Aware Images. IEEE Trans. Im. Proc., in press.

[12] E.P. Simoncelli, E.H. Adelson. Noise Removal via Bayesian Wavelet Coring. Proc. of the 3rd Int. Conf. on Image Proc., I, Laussane, Switzerland, pp. 379-382, Sept. 1996.

[13] J. Portilla, V. Strela, M.J. Wainwright, E.P. Simoncelli. Image Denoising using Scale Mixtures of Gaussians in the Wavelet Domain. IEEE Trans. Image Proc., 12, 11, 1338-51, 2003.

[14] M.J. Fadili, J.L. Starck. EM Algorithm for Sparse Representation-Based Image Inpainting. Int. Conf. Image Proc., 2, pp. 61-63, Genoa, Italy, Sep. 2005.

[15] E.J. Candes, D.L. Donoho. Curvelets: A Surprisingly Effective Nonadaptive Representation for Objects with Edges. Curves and Surfaces IV. P.J. Laurent ed., 1999

[16] D.L. Donoho. For Most Large Undetermined Systems of Linear Equations the Minimal 11-norm Solution is also the Sparsest Solution. Technical Report, September 2004. http://wwwstat.stanford.edu/ donoho/Reports/

[17] J.L. Starck. Morphological Component Analysis. Proc. of the SPIE, 5914, San Diego, CA, Aug. 2005.

[18] D.L. Donoho, M. Elad. Optimally Sparse Representation from Overcomplete Dictionaries vial L1-norm Minimization. Proc. Natl. Acad. Sci., USA, 100, 5, pp. 2197-3002, 4, 2002.

[19] O.G. Guleryuz. On Missing Data Prediction using Sparse Signal Models: A Comparison of Atomic Decomposition with Iterated Denoising. Proc. of the SPIE, 5914, 1G, San Diego, CA, Aug. 2005.

[20] D.C. Youla. Generalized Image Restoration by the Method of Alternating Orthogonal Projections. IEEE Trans. Circuits and Syst., CAS-25, 9, 1978. 